\title{
LA COOPERATION D'ONCOGENES DANS LA TRANSFORMATION CELLULAIRE
}

\author{
Samarut J., Laboratoire dre Biologie Molículaire el Cellulairc, Ecole Normale Suptrieure
} de: 1 you, 46, allée d'thilice 69564 1.yon cédex 07

La transformation oncogène des cellules s'accompagne de modifications dans leur structure et leur physiologie (changements de morphologie, blocage de la différenciation, altérations du cytosquelette et de la matrice extracellulairc, dérèglements de la croissance). Ces modifications affectent à des degrés divers le comportement des cellules transformées, en culture ou in vitro: croissance anarchique indépendante des facteurs régulateurs externes, formation de tumeurs in vivo. Les modeles expérimentaux de la transformation reposent sur l'introduction et l'expression de séquences génétiques oncogènes dans des cellules cibles en culture ou in vivo. Les approches in vivo trouvent une extension nouvelle dans l'utilisation d'animaux transgéniques. Dans la plupart des modèles étudiés, la transformation complète de cellules primaires en cellules tumorales ou leucémiques nécessite l'intervention de plusieurs oncogènes qui agissent en coopération. Ces observations confirment les hypothèses faisant intervenir plusieurs événements génétiques dans le développement de cancers. Ccpendant dans des tumeurs humaines spontanées il n'a pas encore été possible d'identifier des ensembles coopératifs d'oncogènes, et les seuls oncogènes identifiés sont très peu diversifiés entre les différents types de tumeurs. Ces données conduisent à imaginer l'existence d'autres systèmes de gènes impliqués dans la transformation. Parmi ces gènes pourraient se trouver des gènes, les oncogènes récessifs ou anti-oncogènes, dont la fonction normale serait de réprimer la croissance, et dont l'inactivation pourrait contribuer à la transformation.

PROLIFERATION D'HEPATOCYTES DE RAT PAR TRANSFECTION IN VITRO OU TN VIVO A L,'AIDE DE PIASMIDISS ONCOGFNES (Ha-rAS OU BPV) VIIICULES PAR DES I.IPOSOMLS : EXPRLSSION D'UIN ONCOGLNE GT DLE PITENOTYYES METABOLIQULS DU FOLE.

Fischbach M., Cao II., Diez Ihanez M., 'I'saconas C., Alouani S.*, Montandon li., Chessebeuf-Padieu, M., Bromley P.*, Dreano M.*, Padieu P.

Biochinie Médicale, Faculté de Médecine, F-21033 Dijon; (x) Intracel, CH-1227 Carouge-Genève.

Une méthode de transfection cellulaire utilisant un plasmide oncogène, soit le pEJ vecteur du c. onc. Ha-ras muté, soit le plasmide pl7HBN porteur de la séquence transformante du BPV et du gène de l'hGH, encapsulé dans des liposomes a été appliquée à la transfection in vitro d'hépatocytes de rat isolés par la collagénase et à la transfection in vivo du foie d'un rat en injectant par voie intraveineuse la préparation de liposomes. La recombinaison de l'oncogène a permis d'isoler les cellules hépatiques prolifératives d'origine parenchymateuse. L'hybridation par "dot blot" de la sonde 4,3 kpb KpnI du BPV a montré sa recombinaison dans les hépatocytes prolifératifs. L'hormone de croissance humaine (hGH) est sécrétée soit in vivo dans le plasma du rat soit in vitro par les hépatocytes prolifératifs recombinant dans le cas de la transfection par le p17HBN ou d'une co-transfection par les plasmides pEJ et p17hGHneo. Les 1ignées d'hépatocytes prolifératifs résultant de la recombinaison du Ha-ras ont gardé après plusieurs mois de culture la capacité d'exprimer des fonctions propres au foie et au même niveau d'activité : synthèse des acides biliaires primaires libres et conjugués, sécrétion d'albumine et de transferrine, et expression des mono-oxygénases à cytochrome $\mathrm{P}-450$ métabolisant un procancérogène, le 2-acétylaminofluorène, en métabolites aryl hydroxylés et surtout en son cancérogène proche, le N-bydroxy-2-acétylaminofluorène. En conclusion, la recombinaison d'un plasmide oncogène dans des hépatocytes, quiescents par nature, se traduit par une reactivation de leur cycle mitotique sans perte par la lignée d'hépatocytes de phénotypes métaboliques du foie. Enfin, la co-transfection avec un gène codant pour une proteine exogène se traduit par son expression dans la lignée recombinée transtormée. 